\title{
Input Impedance of Rectangular Microstrip Antennas on Spherical Bodies using MoM and Attachment Modes
}

\author{
Leonardo A. Costa, Odilon M. C. Pereira-Filho*, and Fernando J. S. \\ Moreira \\ Federal University of Minas Gerais, Brazil, www.delt.ufmg.br
}

\section{Introduction}

Microstrip antennas are attractive for their low cost, low profile and conformal characteristics. The use of such kind of antennas mounted on spherical bodies can be demonstrated in aeronautics and military areas. Previous works on microstrip antennas on spherical bodies most often used approximated methods, like cavity method [1], and electric surface current method [2]. The input impedance was usually determined using the effective loss tangent of the cavity [3] or the generalized transmission line method [4]. In [5] the Moment method is used in a spherical annular microstrip antenna but only the harmonic modes on the path were used to compute the input impedance. In [6] a spherical rectangular microstrip antenna is analyzed using just one harmonic mode, while the probe self impedance is not taken into account in the impedance calculation. In this work ${ }^{1}$ we perform a full-wave analysis of spherical rectangular microstrip antennas using method of moments. We also introduce an attachment current [7], which avoids the singularity caused by the probe and assures the continuity of the current in the probe/patch connection. The use of the attachment mode reduces the number of basis functions, speeding up the calculations.

\section{Theoretical Formulation}

The geometry of the spherical rectangular microstrip antenna is shown in fig. 1. A perfect electric conducting sphere with radius $r_{1}$ is covered by a dielectric layer with thickness $\delta r$ and relative permittivity $\epsilon_{r}$. A patch of perfect electric conductor is placed on the dielectric layer, limited by the angles $\theta_{1}$ and $\theta_{2}\left(\theta_{2}=\theta_{1}+\delta \theta\right)$, and by $\phi_{1}$ and $\phi_{2}\left(\phi_{2}=\phi_{1}+\delta \phi\right)$, in spherical coordinates. The antenna is fed by a coaxial line with a known radial current $\vec{J}_{\text {probe }}$, as the dielectric layer has a small thickness compared to the wavelength in the dielectric. The probe is formed by four radially directed surface currents placed on the side walls of a spherical sector limited by the angles $\theta_{f 1}$ and $\theta_{f 2}$, and by $\phi_{f 1}$ and $\phi_{f 2}$, in spherical coordinates.

\section{Attachment Current and Method of Moments}

As mentioned above the use of attachment currents [7] assures the continuity of the feed current, avoiding the otherwise resulting singularity. In order to certify that the current is continuous at the connection region, we introduced an attachment current $\vec{J}_{\text {att }}$ formed by four exponentially decaying surface currents, one for each side of the probe, and limited by the microstrip boundaries. Now, the current formed by the

\footnotetext{
${ }^{1}$ This work was partially supported by CNPq.
} 


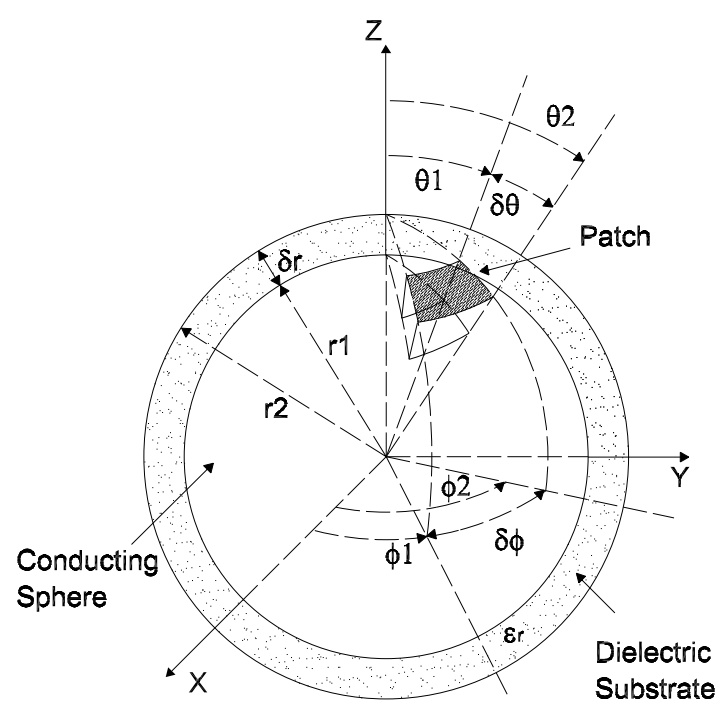

Figure 1: Geometry of Spherical-rectangular Microstrip Antenna

sum of the attachment current and probe current, called $\vec{J}_{s}$, is continuous and the near field does not diverge.

The patch current is written as the sum of the attachment current and a set of sinusoidal basis functions, which amplitudes are to be determined, following the method of moments [8]. The integral equation results from imposing the boundary conditions at the microstrip surface. Applying the Galerkin's procedure, the basis functions are used as test functions generating a matrix problem. The solutions of this problem are the basis current amplitudes. Through the normalized FourierLegendre Transform [6], which is applied over the currents and fields on the surface $r=r_{2}$ the elements of the Impedance matriz and Voltage vector in the method of moments can be obtained and they are respectively given by:

$$
\begin{gathered}
\mathcal{Z}=r_{2}^{2} \sum_{n=1}^{\infty} \sum_{m=-n}^{m=n}\left[\tilde{\mathbb{J}}_{\text {test }}(n,-m)\right]^{T} \overline{\overline{\mathbb{G}_{t}^{E}}}\left(n, r_{2}\right) \tilde{\mathbb{J}}_{\text {basis }}(n, m) \\
\mathcal{V}=-r_{2}^{2} \sum_{n=1}^{\infty} \sum_{m=-n}^{m=n}\left[\tilde{\mathbb{J}}_{\text {test }}(n,-m)\right]^{T} \overline{\overline{\mathbb{G}_{t}^{E}}}\left(n, r_{2}\right)\left[\tilde{\mathbb{J}}_{\text {eqprobe }}(n, m)+\tilde{\mathbb{J}}_{\text {att }}(n, m)\right]
\end{gathered}
$$

where the matrix $\overline{\overline{\mathbb{G}_{t}^{E}}}\left(n, r_{2}\right)$ is the Green's function in the transform domain [2] that relates the tangential electric field with the surface electric currents, both on $r=r_{2}$. And $\tilde{\mathbb{J}}_{\text {eqprobe }}(n, m)$ is the transform of the equivalent surface current in $r=r_{2}$, which produces in the free space the same field produced by the probe current. 


\section{Input Impedance}

The input impedance of the antenna is given through the reciprocity by:

$$
Z_{\text {in }}=-\frac{1}{I^{2}} \int_{V^{\prime}}\left[\vec{E}\left(\vec{J}_{\text {basis }}^{\text {total }}\right)+\vec{E}\left(\vec{J}_{\text {probe }}\right)+\vec{E}\left(\vec{J}_{\text {att }}\right)\right] \bullet \vec{J}_{\text {probe }} \mathrm{d} v^{\prime}
$$

The portions of $Z_{i n}$ corresponding to $\vec{J}_{\text {probe }}$ and $\vec{J}_{\text {att }}$ are individually divergent. This is caused by charge accumulations at the connection probe/microstrip. An alternative to overcome these singularities is to introduce currents with the same singular characteristic without changing the original problem. A singular current is obtained when the microstrip is replaced by a perfect conducting shield covering the entire dielectric layer. This structure represent a probe excited spherical cavity. The induced current on the shield surface, called $\vec{J}_{c}$, can be added and subtracted as follows:

$$
Z_{\text {in }}=-\frac{1}{I^{2}} \int_{V^{\prime}}\left[\vec{E}\left(\vec{J}_{\text {basis }}^{t o t a l}\right)+\vec{E}\left(\vec{J}_{\text {probe }}\right)+\vec{E}\left(\vec{J}_{c}\right)+\vec{E}\left(\vec{J}_{\text {att }}\right)+\vec{E}\left(-\vec{J}_{c}\right)\right] \bullet \vec{J}_{\text {probe }} \mathrm{d} v^{\prime}
$$

Now we can identify individually convergent terms:

$$
\begin{aligned}
Z_{\text {cav }} & =-\frac{1}{I^{2}} \int_{V^{\prime}}\left[\vec{E}\left(\vec{J}_{\text {probe }}\right)+\vec{E}\left(\vec{J}_{c}\right)\right] \bullet \vec{J}_{\text {probe }} \mathrm{d} v^{\prime} \\
Z_{D 1} & =-\frac{1}{I^{2}} \int_{V^{\prime}}\left[\vec{E}\left(\vec{J}_{\text {att }}\right)+\vec{E}\left(-\vec{J}_{c}\right)\right] \bullet \vec{J}_{\text {probe }} \mathrm{d} v^{\prime}
\end{aligned}
$$

\section{Results and Conclusions}

Fig.2 compares the results obtained using the present formulation with those previously shown in [6]. The patch can be considered rectangular with dimensions $2.5 \mathrm{~cm}$ (in $\hat{\theta}$ direction) and $4.0 \mathrm{~cm}$ (in $\hat{\phi}$ direction) and centered on the XY plane. The radial dimensions are $r_{1}=10.0 \mathrm{~cm}$ and $\delta_{r}=1.58 \mathrm{~mm}$. The probe is centered in $\hat{\phi}$ direction, but $1.1 \mathrm{~cm}$ out of the center in $\hat{\theta}$ direction. We can observe that the resonances occur in different frequencies, caused by the more accurate analysis, which was done here. We have used 100 base functions on each direction, while [6] has used only one base function.

This work presented a full-wave analysis of a spherical rectangular microstrip antenna using method of moments, emphasizing the use of an attaching current in the modeling of the pach currents and calculation of its input impedance.

\section{References}

[1] K.Y.Wu and J.F.Kauffman, "Radiation Pattern for Spherical-Rectangular Microstrip Antennas", IEEE Antennas Propagat. Int. Symposium Digest, 1983, pp. 43-46. 


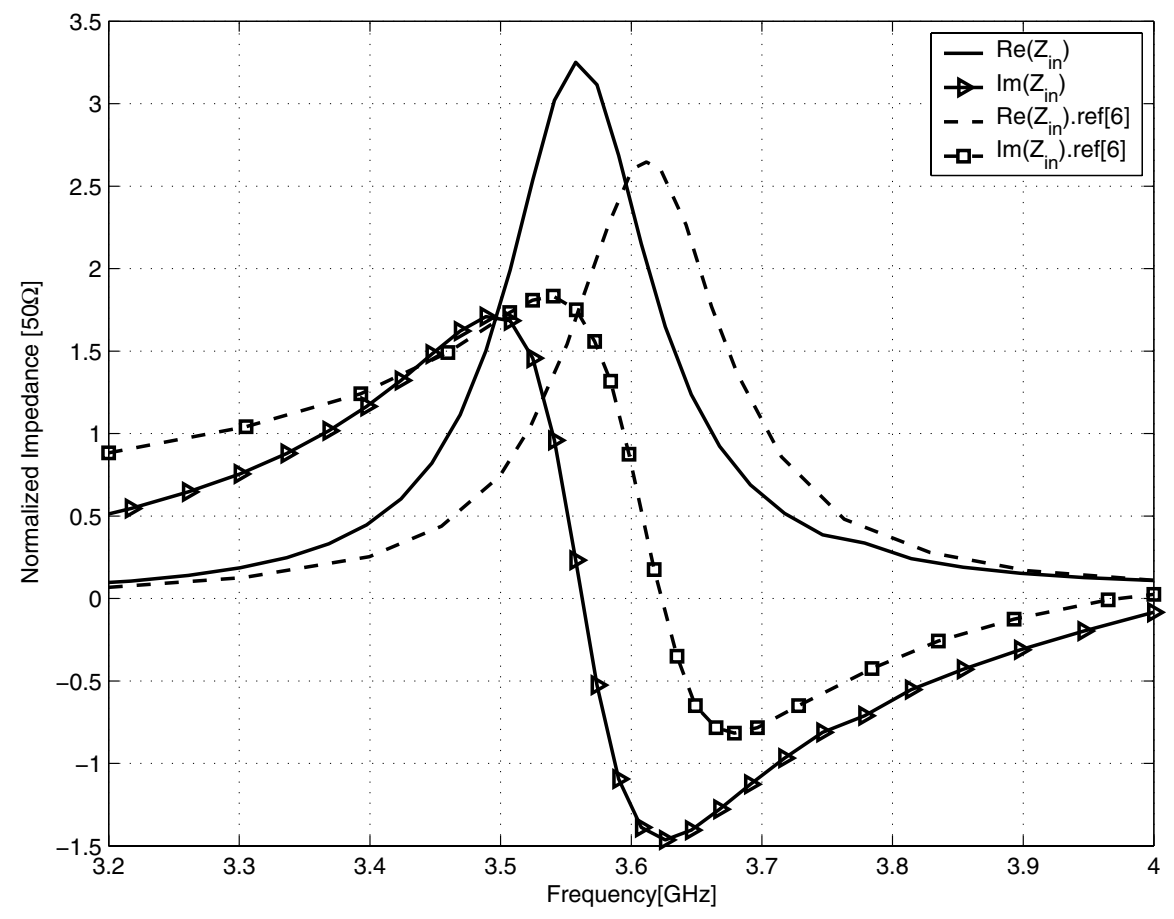

Figure 2: Input Impedance - Rectangular Patch [6] with $2.5 \mathrm{~cm} \times 4.0 \mathrm{~cm}$ centered on XY plane, $r_{1}=10 \mathrm{~cm}$ and $\delta_{r}=1.58 \mathrm{~mm}$, probe $1.1 \mathrm{~cm}$ out of the patch center

[2] W.Y.Tam and K.M.Luk, "Far field analysis of spherical-circular microstrip antennas by electric surface current models", IEEE Proc.-H. vol. 138, No.1,February 1991, pp. 98-102.

[3] K.M.Luk, K.F.Lee and J.S.Dahele, "Analysis of the Cylindrical-Rectangular Patch Antenna", IEEE Trans. Antennas Propagat., vol.37, No.2 , Feb. 1989, pp. 143-147.

[4] B.Ke and A.A.Kishk, "Analysis of spherical circular microstrip Antennas", IEEE Proc.-H. vol. 138, No.6, Dec. 1991, pp. 542-548.

[5] W.Y.Tam, A.K.Y.Lai and K.M.Luk, "Input Impedance of spherical microstrip antenna", IEEE Proc. Microw. Antennas Propagat., vol. 142, No.3,June 1995, pp. 285-288.

[6] N.Burum and Z.Sipus, "Input Impedance and Mutual Coupling of Spherical Rectangular Microstrip Patch Antennas", Procedings of ICECom 2003, Dubrovnik, Croatia, Oct. 2003 pp. 209-212.

[7] A.N.Tulintseff, S.M.Ali and J.A.Kong, "Input Impedance of a Probe-Fed Stacked Circular Microstrip Antennas", IEEE Trans. Antennas Propagat., vol.39, No.3 , March 1991, pp. 381-390.

[8] R.F.Harrington, "Field Computation by Moment Method", Macmillian, New York, 1968. 